\title{
Convergencia y desarrollo en la Argentina urbana (2003-2016)
}

\author{
Fernando Antonio Ignacio González, \\ Maria Emma Santos, Silvia London
}

\begin{abstract}
Resumen: Este articulo analiza la evolución reciente de las disparidades territoriales en Argentina, medida por un Índice de Pobreza Multidimensional, mediante un análisis formal de convergencia entre aglomerados. En particular, se testea la existencia de convergencia- $\beta$ absoluta y convergencia- $\sigma$. La información proviene de los microdatos de la Encuesta Permanente de Hogares. Los resultados sugieren que, si bien en un análisis punta-a-punta del período se observa una reducción de la pobreza acompañada de una reducción de las disparidades territoriales entre los principales aglomerados urbanos de Argentina, al desagregar por subperíodos, se evidencia que en los períodos de crecimiento económico (esencialmente del 2003 al 2009) se incrementaron las disparidades territoriales. En tanto que en los periodos de estancamiento, estas disparidades se redujeron.
\end{abstract}

Palabras clave: crecimiento económico, disparidades territoriales, hogares, pobreza multidimensional

\section{Introducción}

Una renovada preocupación se ha extendido en años recientes sobre la cuestión de la desigualdad a escala global (Atkinson, 2015; Piketty, 2014). Esto es especialmente relevante considerando que elevados niveles de desigualdad se asocian a mayor disrupción social (Alesina \& Perotti, 1996), inestabilidad económica y financiera (FMI, 2015) y una ineficiente utilización de los recursos (Krugman, 2012).

La evidencia empírica sugiere que la desigualdad entre países ricos y pobres, en términos de ingresos, ha disminuido en las últimas décadas (Qureshi, 2017)-especialmente traccionada por las dinámicas de China e India-. Sin embargo, la desigualdad dentro de cada país parece estar incrementándose (OCDE, 2015). En este contexto, Latinoamérica aparece 
como la región más desigual del mundo, junto a África subsahariana (Alvaredo \& Gasparini, 2013).

En particular, Argentina es un país con profundas disparidades territoriales. Las provincias del norte argentino han presentado históricamente peores indicadores de desarrollo y bienestar en relación a las demás regiones. El Norte Argentino comprende a las regiones del Noreste ([NEA], incluye a Misiones, Corrientes, Chaco y Formosa) y Noroeste ([NOA], incluye a Salta, Jujuy, Catamarca, Tucumán, La Rioja, Santiago del Estero y Catamarca). ${ }^{1}$ En efecto, el NEA, con el 9 por ciento de la población, aporta apenas 3.7 por ciento del PBI, 1.7 por ciento del total de exportaciones, y sólo el 4.4 por ciento del total de empleos registrados (Tabla 6 del Anexo). Las participaciones del NOA son algo mejores, pero también desproporcionadas en relación con su contribución poblacional. Las tasas de mortalidad infantil del NEA y NOA son dos a tres puntos más altas que en las demás regiones, y el porcentaje de población con necesidades básicas insatisfechas (NBI) es aproximadamente el doble que en el resto de las regiones (Tabla 7 del Anexo). Los recursos destinados a salud también presentan una amplia heterogeneidad regional (Tabla 8 del Anexo).

Considerando la existencia de amplias diferencias en el grado de desarrollo, diversos trabajos han avanzado en el examen formal de las disparidades territoriales en Argentina realizando análisis de convergencia. Al respecto existen tres posibles especificaciones: convergencia- $\beta$ absoluta, convergencia- $\beta$ condicional o convergencia- $\sigma$. Estos antecedentes tienden a considerar la dinámica del PBI per cápita entre provincias o departamentos y, en general, rechazan la hipótesis de convergencia- $\beta$ absoluta, aunque aceptan la de convergencia- $\beta$ condicional. Con menor frecuencia ha sido explorada la hipótesis de convergencia- $\sigma$ (Utrera \& Koroch, 1998) o de convergencia en indicadores de bienestar distintos a los ingresos (Capello et al., 2013).

En este contexto, este trabajo analiza la evolución de las disparidades territoriales recientes en Argentina (2003-2016). En particular, intentamos responder el siguiente interrogante: ¿Cuál ha sido la relación entre crecimiento económico y disparidades territoriales de pobreza en Argentina? Este trabajo constituye un aporte a la literatura interesada en examinar la relación entre disparidades territoriales de pobreza y crecimiento, para el caso argentino, desde un enfoque multidimensional. El trabajo se encuadra dentro de aquella literatura que explora la relación entre crecimiento y desigualdad (Brida et al., 2020), pero especialmente en aquella que concibe a la pobreza como un fenómeno multidimensional (Alkire \& Santos, 2010; Santos et al., 2019). Esto implica que ser pobre no se limita a la falta de ingresos, sino que incluye múltiples dimensiones del espacio de bienestar (educación, acceso a servicios, vivienda y empleo y seguridad so- 
cial). Esta concepción, a su vez, posee una estrecha relación con el enfoque de las capacidades de Amartya Sen $(1985,2009)$ donde se resalta la importancia de medir el bienestar (y por ende la pobreza) en el "espacio de las capacidades" (no en el de la utilidad, o los recursos), evaluando lo que el individuo puede "hacer" o "ser", o bien en el plano de las privaciones, lo que el individuo "no hace" o "no es" porque no puede, aunque valora y tiene razones para valorar.

Considerando lo anterior, procedemos con un análisis formal de convergencia entre aglomerados urbanos en un Índice de Pobreza Multidimensional para Argentina, el cual incluye las dimensiones de ingresos, vivienda, servicios básicos, educación y empleo y seguridad social. El período bajo estudio inicia en un momento en el que aún estaban muy presentes los efectos inmediatos de una profunda crisis económica y social de Argentina (2001). En efecto, en el primer semestre de 2003 la tasa de desocupación alcanzaba el 20 por ciento y el 54.7 por ciento de la población vivía con ingresos por debajo de la línea de pobreza (INDEC, 2003). A partir de ese año y hasta 2007 se experimenta un rápido crecimiento económico, con un 8.6 por ciento promedio anual de expansión del PBI (INDEC, 2018) acompañado de reducciones en los indicadores de pobreza y mejoras en el nivel de empleo. Sin embargo, a partir de 2009, el crecimiento económico se reduce y durante el período 2011-2015 la tasa de expansión anual sería de un magro 1.7 por ciento. A fines de 2015, se produce un cambio de gobierno y de signo político, ${ }^{2}$ en un contexto de recesión con inflación creciente.

En adelante, la siguiente sección presenta las principales definiciones de cada tipo de convergencia y antecedentes para el caso argentino. La sección posterior describe la metodología de medición de la pobreza multidimensional y de medición de convergencia, así como las fuentes de información empleadas. Luego presentamos los resultados de este trabajo y, finalmente, la última sección discute las principales conclusiones.

\section{Tipos de convergencia y evidencia previa para Argentina}

Un acotado grupo de trabajos analiza las trayectorias económicas provinciales, en términos de crecimiento económico, para indagar respecto a la existencia de convergencia regional. Dos tipos de convergencia son frecuentemente considerados: convergencia- $\beta$ absoluta y convergencia- $\beta$ condicional.

La hipótesis de "convergencia absoluta" sugiere que en el largo plazo el producto per cápita converge a una "misma senda de crecimiento en todas las provincias o regiones". Como resultado, todas las provincias 
convergen a un mismo nivel de producto per cápita, lo cual requiere que las provincias más pobres experimenten mayores tasas de crecimiento. De verificarse esto, los niveles de pobreza convergerían a niveles similares.

La hipótesis de "convergencia condicional", en cambio, sostiene que la convergencia tiene lugar condicionada por un conjunto de características estructurales de cada región, como tasa de analfabetismo, ratio inversión/ PBI, esperanza de vida, entre otras variables que pueden considerarse. Por ende, "el ingreso per cápita converge a una senda de crecimiento específica de cada región" y aquellas que inicien con un nivel de ingreso más alejado de aquel correspondiente a su senda de largo plazo, crecerán más rápidamente.

La convergencia condicional es una condición necesaria pero no suficiente para la convergencia absoluta. Un tercer tipo de análisis, radica en la "convergencia- $\sigma$ ", esto es, una "reducción en la dispersión de los niveles de ingreso per cápita entre provincias", medido por el desvío estándar o coeficiente de variación del logaritmo del producto per cápita (Bernard \& Jones, 1996).

El primer antecedente observado en el tópico de convergencia según el período examinado corresponde a William Maloney y Felipe Valencia Caicedo (2012), quienes analizan la persistencia (o reversión) de la actividad económica regional en América en los últimos quinientos años empleando datos de población e ingresos para el presente, así como datos de población de 1492. Encuentran que aquellas zonas densamente pobladas en la etapa precolonial ${ }^{3}$ son también, en la actualidad, las regiones con mayor densidad poblacional e ingresos per cápita. Lo anterior sugiere que, dentro de cada país, la actividad económica muestra patrones regionales persistentes contrarios a la idea de convergencia.

Sin embargo, los autores encuentran que el caso de Argentina se aparta de las conclusiones generales. Argentina exhibe una correlación negativa entre la densidad poblacional regional en la etapa precolonial y la densidad e ingresos en la actualidad. De tal forma, Misiones y Corrientes-con climas húmedos y subtropicales-eran las áreas más densamente pobladas en la etapa precolonial, mientras que en la actualidad las provincias más pobladas y de mayores ingresos son Buenos Aires, Córdoba, Santa Fe, Entre Ríos y Ciudad de Buenos Aires. Otras provincias de ingresos altos en la actualidad, pero con baja densidad poblacional en la etapa precolonial son Chubut y Santa Cruz.

La reversión en el caso de las provincias patagónicas, explican los autores, se debió a que son provincias con un clima poco atractivo (lo cual afectó negativamente a la cantidad de asentamientos humanos en la etapa precolonial) y abundancia de recursos naturales (lo cual favoreció el establecimiento en períodos posteriores). En el caso de las provincias de la región Centro la reversión se debió a factores idiosincráticos. Maloney y 
Valencia Caicedo resaltan que la creación del Virreinato del Rio de la Plata y la re-orientación obligatoria de los flujos comerciales-especialmente el comercio de metales desde Lima a España - vía el puerto de Buenos Aires explican la reversión en los patrones de densidad poblacional e ingresos. Finalmente, en los casos de Misiones y Corrientes, la reversión habría operado a partir de la relativa abundancia de nativos, lo cual favoreció la creación de "instituciones extractivas" ${ }^{4}$ que afectan negativamente al desarrollo económico, en consonancia con resultados de Daron Acemoglu, Simon Johnson, y James Robinson (2002). Así, la reversión de la actividad económica en Argentina benefició a Buenos Aires y la región Centro, mientras que operó en detrimento de las provincias del NEA.

La Tabla 1 resume los resultados de los estudios empíricos sobre convergencia para provincias argentinas - en orden cronológico según el inicio del período analizado-abarcando desde fines del siglo XIX. La mayor parte de los antecedentes no encuentra evidencia de convergencia absoluta entre provincias, a excepción de María Florencia Aráoz y Esteban Nicolini (2015) para el período 1895-1914 y Mauricio Talassino (2017) para el período 1953-1959. A su vez, con una menor cantidad de estimaciones, los antecedentes son concordantes con la idea de convergencia condicional, salvo Guido Gustavo Porto (1994) para el período 1953-1980. Estimaciones de convergencia- $\sigma$ son realizadas en Gastón Utrera y Javier Koroch (1998) aunque sin resultados concluyentes.

Debe considerarse que los resultados presentados en la Tabla 1 no son comparables. En primer lugar, se refieren a diferentes períodos de tiempo,

Tabla 1 - Estimaciones de convergencia en PBI entre provincias de Argentina

\begin{tabular}{|c|c|c|c|}
\hline Autor & $\begin{array}{l}\text { Período } \\
\text { analizado }\end{array}$ & $\begin{array}{l}\text { Convergencia- } \beta \\
\text { absoluta }\end{array}$ & $\begin{array}{l}\text { Convergencia- } \beta \\
\text { condicional }\end{array}$ \\
\hline Aráoz y Nicolini (2016) & $1895-1914$ & No se rechaza & - \\
\hline Aráoz y Nicolini (2015) & 1914-1953 & Se rechaza & - \\
\hline Talassino (2015) & 1914-1953 & Se rechaza & - \\
\hline Talassino (2017) & 1953-1959 & No se rechaza & - \\
\hline Porto (1994) & 1953-1980 & Se rechaza & Se rechaza \\
\hline Utrera y Koroch (1998) & 1953-1994 & Se rechaza & No se rechaza \\
\hline $\begin{array}{l}\text { Brida, Garrido y } \\
\text { London (2013) }\end{array}$ & 1961-2000 & Se rechaza & - \\
\hline Garrido, et al. (2001) & 1970-1995 & Se rechaza & No se rechaza \\
\hline Figueras, et al. (2014) & 1970-2006 & Se rechaza ${ }^{a}$ & No se rechaza \\
\hline
\end{tabular}

Fuente: elaboración propia

a Para el sub-período 1990-2000 no se rechaza la hipótesis de convergencia absoluta. 
emplean diferentes fuentes de información y utilizan distintos métodos de estimación. Así, Talassino (2017) no encuentra evidencia de convergencia absoluta al estimar por mínimos cuadrados ordinarios - al igual que la mayor parte de los estudios-. Luego, al incorporar la posibilidad de correlación espacial entre observaciones, no rechaza la hipótesis de convergencia absoluta. Este grupo de antecedentes brinda, sin embargo, un panorama de la evolución de las disparidades regionales en Argentina.

Algunas conclusiones interesantes pueden ser resaltadas. Aráoz y Nicolini (2016, p. 18) afirman que, el conjunto de políticas económicas que implicó pasar de una economía más abierta, con bajos niveles de intervención estatal en 1914, a una más elevada intervención estatal orientada al mercado interno en 1953, no produjo ningún cambio significativo en la posición relativa de las provincias argentinas. Lo anterior resulta especialmente relevante sugiriendo la inefectividad de las políticas de "crecer hacia adentro" para reducir las disparidades territoriales.

A su vez, Alberto Jose Figueras, Daniela Cristina, Valeria Blanco, Iván Iturralde y Marcelo Capello (2014) sostienen que las transferencias fiscales hacia las provincias retrasan la convergencia, argumentando la posible existencia de una "enfermedad holandesa". Aquellas jurisdicciones que reciben mayores transferencias por habitante tienden a destinar una mayor cantidad de recursos a contratación del factor trabajo (empleo público), generando un incremento en la prima salarial del sector público, especialmente entre trabajadores de baja calificación, en relación al sector privado. Este incremento crea una presión salarial en el mercado laboral y resiente a aquellos sectores intensivos en mano de obra de baja calificación $\mathrm{y}$, en última instancia, al producto provincial.

Por otra parte, utilizando información censal y del Ministerio de Salud, Marcelo Capello, Alberto Figueras, Sebastian Freille y Pedro Moncarz (2013) reportan la existencia de convergencia condicional entre regiones para el período 1970-2001 en los indicadores de escolarización, analfabetismo, NBI, esperanza de vida, mortalidad, calidad de la vivienda, tenencia y hacinamiento. Resaltan que, a pesar de la inexistencia de convergencia absoluta en el ingreso per cápita, al menos se observa convergencia en los indicadores de bienestar seleccionados. Lo anterior es relevante dada su coincidencia con los indicadores empleados en la medición de la pobreza multidimensional.

\section{Metodología y fuente de información}

A continuación, el apartado siguiente describe la fuente de información empleada, el apartado posterior detalla la metodología de cómputo del 
índice de pobreza multidimensional y finalmente, el último apartado presenta la metodología de análisis de convergencia.

\section{Fuentes de información}

Utilizamos los microdatos provistos por el Instituto Nacional de Estadísticas y Censos (INDEC) recabados por medio de la Encuesta Permanente de Hogares (EPH) en forma trimestral para 31 aglomerados urbanos del país. La EPH es representativa de estas áreas urbanas en la cuales residían, en 2016, 27,201,000 personas (INDEC, 2016a). Considerando que las proyecciones poblacionales de INDEC sugieren para 2016 una población total de 43,590,368, la EPH resulta representativa del 62 por ciento del total poblacional. Si se asume que la proporción de población urbana se mantuvo constante desde el último censo 91 por ciento en 2010-la EPH es, a su vez, representativa del 69 por ciento de la población urbana argentina. El análisis se centra en el período 2003-2016 aunque algunos trimestres no pudieron ser incluidos por falta de datos, a saber: los trimestres I y II del año 2003, III del 2007, III y IV del 2015 y I del $2016 .{ }^{5}$ Esto es una limitación que surge de la fuente de información utilizada y que puede afectar las estimaciones.

Los aglomerados urbanos, del Norte Argentino, relevados en la EPH son: Posadas (Misiones), Gran Resistencia (Chaco), Corrientes, Formosa, Santiago del Estero-La Banda, Jujuy-Palpalá, Gran Catamarca, Salta, La Rioja y Gran Tucumán-Tafí Viejo, todos capitales de las provincias. Esto mismo ocurre con los aglomerados de las demás provincias argentinas, a excepción de Córdoba, Santa Fe, Entre Ríos y Chubut en donde se releva un aglomerado adicional, además de la capital provincial correspondiente, y Buenos Aires, provincia en la que se relevan cinco aglomerados urbanos. ${ }^{6}$ De esta forma, quedan excluidas de la EPH tanto los aglomerados más pequeños como las zonas rurales del país.

Cabe aclarar que, de acuerdo con lo publicado por INDEC (2016b), los resultados de las estimaciones obtenidas para los años 2007 a 2015 deben considerarse con reservas debido a que la encuesta estuvo sujeta a un manejo discrecional en algunos aspectos que afectan la confiabilidad de las estimaciones. ${ }^{7}$

Por último, la serie de Producto Bruto Interno per cápita es aquella que surge de Banco Mundial (2020). Se considera la serie de pesos constantes.

\section{Índice de pobreza multidimensional para Argentina}

A partir de las propuestas de Maria Emma Santos, Pablo Villatoro, Xavier Mancero y Pascual Gerstenfeld (2015) y Santos y Villatoro (2018) em- 
pleamos el Índice de Pobreza Multidimensional para América Latina con adaptaciones para el caso argentino y el cual considera las dimensiones de vivienda, educación, servicios básicos, estándar de vida y empleo y seguridad social. En cada caso evaluamos el logro de los hogares para determinar la existencia de privación (logro por debajo del umbral de privación). Cada indicador consiste en una variable binaria (valor 0 si el hogar no presenta privación y 1 en otro caso). Los indicadores utilizados son:

Tabla 2 - Dimensiones, indicadores y ponderaciones

\begin{tabular}{lll}
\hline Dimensiones & Indicador de privación & Ponderación \\
\hline $\mathbf{1 .}$ Vivienda & & $\mathbf{2 2 . 2 \%}$ \\
\hline $\begin{array}{l}\text { Materiales de la } \\
\text { vivienda }^{a}\end{array}$ & $\begin{array}{l}\text { Hogares con piso de tierra o materiales } \\
\text { de techo o paredes precarios (residuos, } \\
\text { cartón, caña, paja, otros). }\end{array}$ & $\mathbf{7 . 4 \%}$ \\
\hline Personas por cuarto & $\begin{array}{l}\text { Hogares con 3 o más personas por } \\
\text { cuarto. }\end{array}$ & $\mathbf{7 . 4 \%}$ \\
\hline $\begin{array}{l}\text { Tenencia de la } \\
\text { Vivienda }\end{array}$ & $\begin{array}{l}\text { Hogares que viven en casas ocupadas } \\
\text { ilegalmente o en una casa cedida o } \\
\text { prestada. }\end{array}$ & $7.4 \%$ \\
\hline
\end{tabular}

\section{Servicios Básicos}

$22.2 \%$

\begin{tabular}{|c|c|c|}
\hline $\begin{array}{l}\text { Agua de Fuente } \\
\text { Mejorada }^{b}\end{array}$ & $\begin{array}{l}\text { Hogares con agua de red fuera del } \\
\text { terreno, agua de pozo sin bomba mecá- } \\
\text { nica, agua embotellada, agua de lluvia, } \\
\text { rio o arroyo. }\end{array}$ & $7.4 \%$ \\
\hline Sanidad Mejorada ${ }^{b}$ & $\begin{array}{l}\text { Hogares } \sin \text { baño, baño compartido o } \\
\text { letrina sin fosa séptica. }\end{array}$ & $7.4 \%$ \\
\hline Energíac $^{c}$ & $\begin{array}{l}\text { Hogares que utilizan madera, carbón } \\
\text { o estiércol como combustible para } \\
\text { cocinar. }\end{array}$ & $7.4 \%$ \\
\hline 3. Educación & & $22.2 \%$ \\
\hline $\begin{array}{l}\text { Asistencia a la } \\
\text { escuela }\end{array}$ & $\begin{array}{l}\text { Hogares donde al menos un chico de } \\
\text { entre } 6 \text { y } 17 \text { años no asiste a la escuela. }\end{array}$ & $7.4 \%$ \\
\hline Rezago Escolar & $\begin{array}{l}\text { Hogares con al menos un chico de entre } \\
6 \text { y } 17 \text { años atrasado más de } 2 \text { años con } \\
\text { respecto al grado para su edad. }\end{array}$ & $7.4 \%$ \\
\hline Logro Educativo & $\begin{array}{l}\text { Hogares donde ningún miembro de } \\
\text { entre } 20 \text { y } 59 \text { años tiene al menos baja } \\
\text { secundaria completa o ningún } \\
\text { miembro de } 60 \text { años o más tiene } \\
\text { primaria completa. }\end{array}$ & $7.4 \%$ \\
\hline
\end{tabular}




\begin{tabular}{|c|c|c|}
\hline Dimensiones & Indicador de privación & Ponderación \\
\hline $\begin{array}{l}\text { 4. Empleo y } \\
\text { Seguridad Social }\end{array}$ & & $11.1 \%$ \\
\hline Empleo $^{\mathrm{d}}$ & $\begin{array}{l}\text { Hogares con al menos un miembro de } \\
\text { entre } 15 \text { y } 65 \text { años desempleado, } \\
\text { empleado sin pago o desalentado. }\end{array}$ & $7.4 \%$ \\
\hline Seguridad Social & $\begin{array}{l}\text { Hogares donde ningún miembro tiene } \\
\text { cobertura médica o, ningún miembro } \\
\text { contribuye al sistema de seguridad social } \\
\text { o, ninguno percibe ingresos por pensión } \\
\text { o jubilación. }\end{array}$ & $3.7 \%$ \\
\hline 5. Estándar de vida & & $22.2 \%$ \\
\hline Ingresos $^{\mathrm{e}}$ & $\begin{array}{l}\text { Hogares con ingresos por adulto } \\
\text { equivalente insuficientes para cubrir } \\
\text { necesidades alimentarias y no } \\
\text { alimentarias. }\end{array}$ & $22.2 \%$ \\
\hline
\end{tabular}

Fuente: elaboración propia en base a Santos \& Villatoro (2018)

Notas:

${ }^{a}$ La EPH no brinda información sobre el material predominante en las paredes, de manera que este material fue ignorado.

${ }^{\mathrm{b}}$ Agua y sanidad tienen umbrales diferentes en áreas rurales en el IPM-LA, los cuales no son aplicables aquí, puesto que la EPH sólo recolecta datos en aglomerados urbanos.

c El IPM-LA considera también falta de acceso a electricidad, pero puesto que esta información no está disponible en la EPH, sólo se considera el combustible utilizado para cocinar.

${ }^{\mathrm{d}}$ De acuerdo a la información recabada por la EPH, un trabajador desalentado se define como aquel que a la pregunta de por qué motivo no buscó trabajo en los últimos 30 días, respondió la opción "se cansó de buscar trabajo" o la opción "hay poco trabajo en esta época del año".

e El IPM-LA incluye un indicador de bienes durables en la dimensión de estándar de vida, que aquí no ha podido ser incluido por falta de información en la EPH. El IPM-LA utiliza la definición de ingreso per cápita del hogar, en tanto que esta aplicación utiliza la definición de ingreso por adulto equivalente de acuerdo con la última especificación metodológica de INDEC.

El indicador de ingresos merece una aclaración especial debido a las irregularidades estadísticas en las que incurrió INDEC durante el tiempo en el que estuvo intervenido, entre 2007 y 2015. Para este indicador, entre los años 2003-2006, utilizamos como línea de pobreza la canasta básica total computada por el INDEC. Luego, en vistas de que a partir del año 2007 el INDEC comenzó a subestimar la inflación, se procedió de la siguiente forma: entre los años 2007-2008, utilizamos la canasta del INDEC estimada para el cuarto trimestre de 2006 pero ajustándola por la variación en el Índice de Precios al Consumidor de la serie de Inflación Verdadera (PriceStats). A partir del año 2009 empleamos la canasta estimada por la Fundación de Investigaciones Económicas Latinoamericanas (FIEL). 
La estimación del IPM para cada aglomerado se realiza como sigue. Sean $i=1, \ldots, n_{d}$ las personas en cada aglomerado $d, \mathrm{y} j=1, \ldots, D$ los indicadores, cada uno de los cuales recibe una ponderación $w_{j}$, de forma que $\Sigma_{j} w_{j}=1$. Cada indicador de privación $g_{i j}{ }^{0}$ es una variable binaria con valor $w_{j}$ si la personas $i$ se encuentra privada es ese funcionamiento, y 0 en los demás casos. Las ponderaciones y umbrales de privación son los que surgen de la Tabla 2.

Una persona es definida como pobre si su puntaje de privaciones, definido como la suma de los valores de privación, es igual o mayor al umbral de pobreza: $c_{i}=\sum_{j=1}^{11} g_{i j}^{0} \geq k$.

Las privaciones de aquellos no pobres son censuradas. Los indicadores de privación censurados son definidos como: $g_{i j}{ }^{0}(k)=g_{i j}{ }^{0}$ si $c_{i} \geq k, y g_{i j}{ }^{0}(k)$ $=0$ en los demás casos. Así, el IPM para cada aglomerado $d$ es computado como sigue:

$$
M P I_{d}=M_{0 d}=\frac{1}{n} \sum_{i=1}^{n} \sum_{j=1}^{D} g_{i j}{ }^{0}(k)
$$

El IPM es una de las medidas $\left(M_{0}\right)$ de la familia de medidas de pobreza Sabina Alkire y James Foster $(2007,2011)$ y el cual ha presentado una creciente utilización entre académicos y hacedores de política desde la publicación del Índice de Pobreza Multidimensional Global (Alkire \& Santos, 2010). El IPM es la tasa de incidencia (H) (proporción de personas identificadas como multidimensionalmente pobres) ajustada por intensidad de la pobreza (A) (proporción promedio de privaciones que experimentan los pobres). El IPM es estimado, a partir de los microdatos de la EPH, para cada año en el período analizado (2003-2016).

El valor del umbral de pobreza multidimensional $k$ utilizado en estas estimaciones es del 25 por ciento. Es decir, una persona debe estar privada, en al menos, el 25 por ciento de los indicadores ponderados para ser considerada pobre. Esto implica que es necesario presentar privaciones en más de una dimensión completa para ser identificado como pobre. Nótese que dada la estructura de ponderaciones, este requerimiento se cumple cuando se experimentan como mínimo las siguientes combinaciones de privaciones: (a) privación en ingresos y en algún otro indicador (como por ejemplo seguridad social), o (b) privación en los dos indicadores de la dimensión de empleo y seguridad social, y además en otros dos indicadores, o (c) cuando se está privado en toda la dimensión de vivienda o servicios básicos, o educación (o su equivalente, es decir, en tres indicadores con ponderación de 7.4 por ciento) y en cualquier otro indicador adicional. Este umbral de pobreza multidimensional $k$ es un umbral conservador.

La construcción de este IPM implica tomar decisiones normativas sobre qué ponderación recibe cada indicador o cuáles umbrales de priva- 
ción se usarán en cada caso. Lo mismo es cierto para el umbral de pobreza multidimensional $(k)$. Por ello, es relevante conocer cuan robustas son las estimaciones obtenidas ante cambios en alguno de estos parámetros. Al respecto, Santos y Villatoro (2018) encuentran para 17 países de la región, que el IPM de referencia de este trabajo es robusto ante cambios en estos parámetros. En general, más del 80 por ciento de todos los pares de combinaciones país-año son robustos (esto es, una combinación país-año es más/menos pobre que otra bajo cualquier especificación alternativa de IPM). Para el caso del IPM adaptado para Argentina, Fernando Antonio Ignacio González (2019) encuentra que este índice es robusto ante cambios en las ponderaciones de los indicadores y también ante variaciones en el umbral de pobreza multidimensional $(k)$. Así, más del 65 por ciento de los pares de combinaciones aglomerado-año son robustos.

\section{Estrategia de estimación empírica}

El análisis formal de convergencia se lleva a cabo considerando las especificaciones de convergencia- $\beta$ absoluta (más estricta que convergencia- $\beta$ condicional) y de convergencia- $\sigma$ para los 31 aglomerados urbanos incluidos en la EPH para el período 2003-2016.

Para testear la existencia de convergencia- $\beta$ absoluta estimamos la siguiente ecuación:

$\ln \left(\frac{I P M_{i, t}}{I P M_{i, t-1}}\right)=\alpha+\beta \ln \left(I P M_{i, t-1}\right)+\varepsilon_{i, t}$

en donde se espera un $\beta<0$ en caso que haya tenido lugar un proceso de convergencia. A los fines de la estimación, construimos un panel de aglomerados para el período 2003-2016. La velocidad de convergencia - en caso de existir-puede ser calculada a partir de la siguiente ecuación (Ramón-Berjano, 2004):

$V=-\ln \left(\frac{1-\beta T}{T}\right)$

en donde T es el período de tiempo, es decir, el total de años considerados.

Por su parte, para testear la existencia de convergencia- $\sigma$, evaluamos si se satisface la siguiente inecuación:

$\sigma_{t}<\sigma_{t-1}$

donde $\sigma_{t}$ es el desvío estándar del logaritmo natural del índice de pobreza multidimensional en el año $t$. Una especificación fuerte de convergencia- $\sigma$ requiere que la desigualdad 4 se cumpla para todo $t$. Permitiendo la po- 
sibilidad de fluctuaciones en subperíodos, se han propuesto medidas alternativas que consideran el comportamiento asintótico de la covarianza muestral - entre el desvío estándar de la variable de interés y el tiempo-. $\mathrm{Al}$ respecto, empleamos la siguiente forma funcional para testear la existencia de convergencia- $\sigma$ (Kong, Phillips \& Sul, 2017):

$\sigma t=\varnothing+\pi t+\mu_{t}$

donde $t$ es la variable de tiempo del panel - considerando cada uno de los años incluidos $-\mathrm{y}$ es el término de error. Se espera-en caso de existir convergencia- $\sigma$-que el coeficiente sea negativo y significativo.

Por otra parte, resulta conveniente testear la relación entre la existencia de convergencia y crecimiento. En particular utilizamos la siguiente ecuación:

$\sigma_{t}=c+d P B I p c_{t-1, t}+g_{t}$

donde $\sigma_{t}$ es, nuevamente, el desvío estándar del logaritmo natural del IPM para el año $t, P B I p c_{t-1, t}$ es la tasa de crecimiento del PBI per cápita entre $t-1 \mathrm{y} t$, mientras que $g_{t}$ es el término de error. Esta estimación asume que la dinámica de la actividad económica argentina, en el período analizado, refleja adecuadamente los cambios en la actividad económica en los aglomerados urbanos incluidos en la EPH.

\section{Resultados}

\section{Estimaciones del Índice de pobreza multidimensional}

En primer lugar, presentamos los resultados que surgen de estimar el IPM agrupados para regiones de Argentina (en Tabla 9 del Anexo se presentan las estimaciones para cada uno de los 31 aglomerados). Debe tenerse en cuenta que, hacia 2003, Argentina iniciaba su fase de recuperación económica luego de la crisis del 2001 y, por ello, los niveles iniciales de pobreza son elevados para todas las regiones y aglomerados. En consonancia con los antecedentes examinados, el Norte Argentino presenta mayores niveles de pobreza multidimensional. Las regiones de Cuyo y Centro muestran, a lo largo de toda la serie, niveles de pobreza similares entre sí con la región Centro experimentando un pequeño incremento en el último año considerado - terminando con un valor de IPM mayor que en el año 2011 - . Por su parte, la región Sur presenta niveles de pobreza menores a las demás regiones que persisten hacia 2016. 
En términos de su evolución, se observa que entre 2003 y 2008 hubo una clara tendencia a la reducción en los niveles de pobreza multidimensional para todas las regiones, en tanto que a partir de 2009 se registra un estancamiento con fluctuaciones en las regiones de Centro, Cuyo y Sur, en tanto que las regiones del NEA y NOA continuaron disminuyendo la pobreza. Así, punta-a-punta, se observa una reducción de las disparidades entre el Norte y las regiones del Centro y Cuyo, aunque en menor medida respecto al Sur.

Gráfico 1 - Índice de pobreza multidimensional para regiones en Argentina (2003-2016)
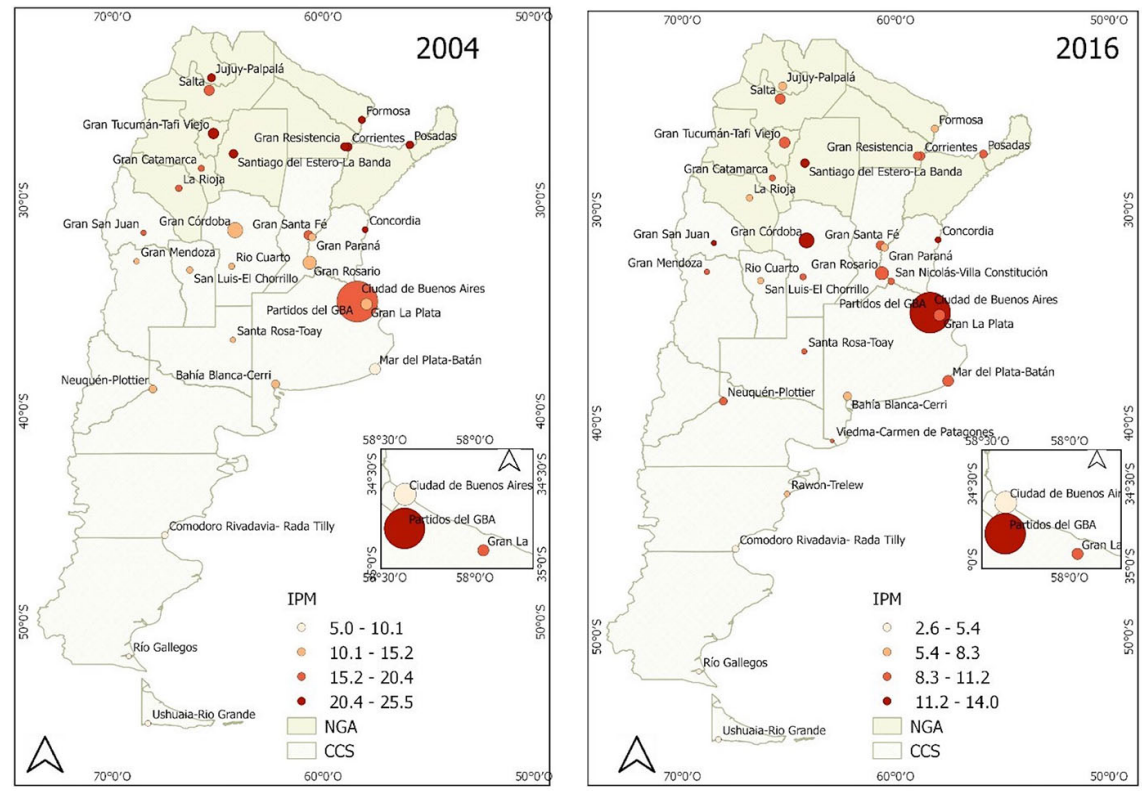

Fuente: González \& Santos (2020)

\section{Análisis de convergencia}

Considerando el panel de 31 aglomerados urbanos relevados en la EPH, los resultados en términos de convergencia- $\beta$ sugieren lo siguiente.

Los resultados de las estimaciones de la Tabla 3 sugieren que, entre 2004-2016, tuvo lugar un proceso de convergencia absoluta entre los principales aglomerados urbanos de la EPH en términos de pobreza multidimensional. Sin embargo, al desagregar en subperíodos se observan comportamientos dispares: hasta 2007 ocurrió un proceso de divergencia, entre 2008-2011 tuvo lugar una dinámica de convergencia, aunque a un menor ritmo que el observado entre 2012-2016. El parámetro de velocidad 
Tabla 3 - Convergencia- $\beta$ absoluta de IPM en aglomerados urbanos de Argentina

\begin{tabular}{lllll}
\hline \multicolumn{5}{c}{ Dependiente: logaritmo del ratio IPM } \\
\hline Variables & $2004-2016$ & $2004-2007$ & $2008-2011$ & $2012-2016$ \\
\hline$\beta$ & $-0.0938^{* * *}$ & $0.0907^{* * *}$ & $-0.0523^{* *}$ & $-0.1721^{* * *}$ \\
\hline Constante & 0.1558 & -0.3836 & 0.0922 & 0.3451 \\
\hline $\mathrm{N}$ & 407 & 119 & 128 & 160 \\
\hline $\mathrm{R}^{2}$ & 0.07 & 0.13 & 0.03 & 0.13 \\
\hline Velocidad & 0.0599 & -- & 0.0475 & 0.1242 \\
\hline
\end{tabular}

* significativo al 10 por ciento, ${ }^{* *}$ significativo al 5 por ciento, ${ }^{* * *}$ significativo al 1 por ciento. Dado que se considera el primer rezago del IPM, se pierde la primera observación de la serie (2003).

Fuente: elaboración propia en base a EPH-INDEC

de convergencia (5.99 por ciento anual) sugiere que, de mantenerse las dinámicas regionales del IPM observadas entre 2004-2016, serían necesarios 11.5 años $^{8}$ más para eliminar el 50 por ciento de la brecha respecto al estado estacionario de pobreza. De tener lugar la dinámica observada entre 2012-2016, se requerirían 5.6 años.

Lo anterior implica, para el caso argentino, que los aglomerados con mayores niveles de pobreza hacia el inicio del período lograron reducirla a un mayor ritmo que aquellos aglomerados con menores niveles iniciales de pobreza. En otras palabras, los aglomerados del Norte Argentino tuvieron una reducción más rápida de la pobreza en relación a sus pares del resto del país. Esto es lo que se observa en el Gráfico 1.

En términos de convergencia- $\sigma$, es posible lograr una primera aproximación a partir de la gráfica del desvío estándar del logaritmo natural del IPM en el tiempo:

Se observa que el desvío estándar muestra una tendencia creciente hasta 2008 - contrario a la idea de convergencia- $\sigma$ y coincidente con la no convergencia- $\beta$-, luego presenta una reducción entre 2008-2012. En adelante la tendencia se mantiene, aunque interrumpida por un significativo aumento en la dispersión en el año 2015. Por lo anterior, no es posible sostener la existencia de convergencia- $\sigma$ en todo el período, tal como plantea la desigualdad (4), dado que no se mantiene para todos los años que el desvío estándar en el año $t$ sea menor al desvío en $t$-1 (aumentos en 2011 y 2015). Al estimar la especificación dada en la ecuación 5-una especificación menos demandante - se obtiene lo siguiente: 
Gráfico 2 • Desvío estándar del logaritmo de IPM (2003-2016)

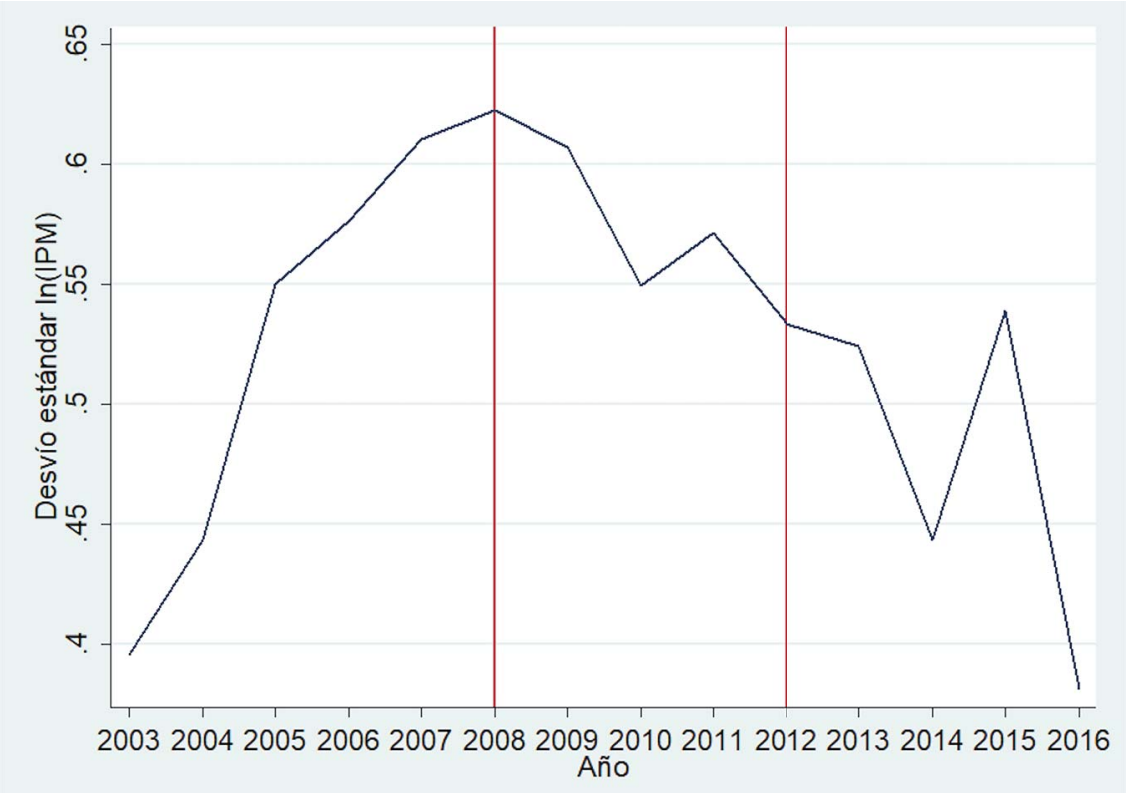

Fuente: elaboración propia en base a EPH-INDEC

Tabla 4 - Convergencia- $ß$ de IPM en aglomerados urbanos de Argentina

\begin{tabular}{lllll}
\hline \multicolumn{5}{c}{ Dependiente: desvío estándar logaritmo IPM } \\
\hline Variables & $2003-2016$ & $2003-2007$ & $2008-2011$ & $2012-2016$ \\
\hline & $-0.0023^{* * *}$ & $0.05637^{* * *}$ & $-0.0210^{* * *}$ & $-0.0290^{* * *}$ \\
& $(0.0009)$ & $(0.0011)$ & $(0.0013)$ & $(0.0026)$ \\
\hline Constante & 5.1640 & -112.5188 & 42.75843 & 58.92993 \\
\hline $\mathrm{N}$ & 448 & 160 & 128 & 160 \\
\hline $\mathrm{R}^{2}$ & 0.02 & 0.02 & 0.67 & 0.43 \\
\hline
\end{tabular}

Fuente: elaboración propia en base a EPH-INDEC

${ }^{*}$ significativo al $10 \%$, ${ }^{* *}$ significativo al $5 \%$, ${ }^{* * *}$ significativo al $1 \%$

Nuevamente, al considerar todo el período se observa la existencia de convergencia, en este caso, en términos de la dispersión de los valores de IPM. A su vez, en el subperíodo 2003-2007, se observa un proceso de polarización (como contraposición a convergencia), en tanto que en los dos subperíodos siguientes se observa convergencia- $\sigma$. Para el subperíodo 
2012-2016 el coeficiente es mayor en valor absoluto indicando que el proceso de reducción de las disparidades tiene lugar a un mayor ritmo, consistente con los resultados obtenidos para la convergencia- $\beta$.

En vistas de los resultados anteriores y lo acontecido en la economía argentina en el período de referencia, se presenta una relación negativa entre crecimiento económico y niveles de pobreza: entre 2003-2007 Argentina experimentó una etapa de rápido crecimiento económico y de reducción de la pobreza. Luego, a partir de 2008, la expansión económica se desaceleró, al igual que la disminución en los niveles de pobreza (Gráfico 3). La relación anterior se encuentra ampliamente estudiada en la literatura de pobreza por ingresos y en menor medida en términos de pobreza multidimensional (Santos et al., 2019).

Gráfico 3 • Evolución del PBI per cápita en Argentina (2003-2016)
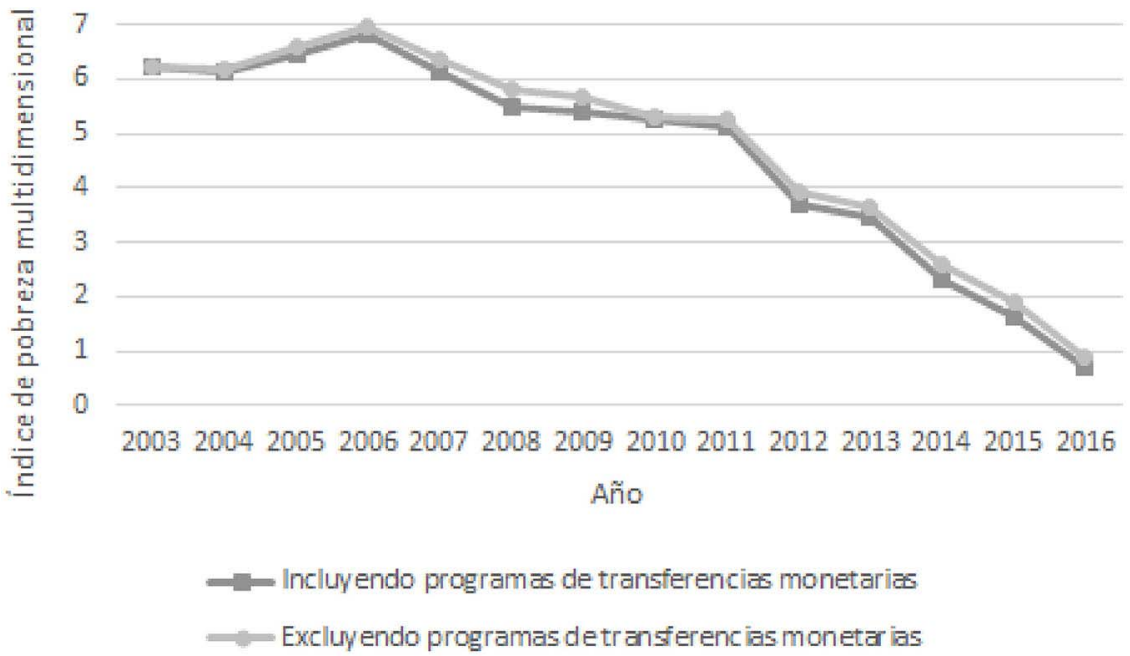

Fuente: elaboración propia en base a Banco Mundial (2020).

Nota: PBI per cápita en pesos constantes

Al mismo tiempo, parece tener lugar una relación negativa entre crecimiento económico y convergencia regional de pobreza. Así, períodos de expansión parecen asociarse a tendencias divergentes de pobreza entre regiones de Argentina, mientras que períodos de estancamiento serían coincidentes con una reducción de las disparidades de pobreza. Esta relación se encuentra menos documentada y parece sugerir la existencia de un dilema á la Kuznets: mayor crecimiento, reducción de la pobreza y aumento de las disparidades regionales o, menor crecimiento, estancamiento en niveles de pobreza y reducción de las disparidades regionales. Esta aparente conflictividad entre objetivos de política eventualmente podría reprodu- 
cirse también a nivel de los países generando una tensión entre el Objetivo de Desarrollo Sostenible de "poner fin a la pobreza en todas sus formas en todo el mundo" (ODS 1) y el de "reducir la desigualdad en y entre los países" (ODS 10).

Por último, se examina la relación entre crecimiento del PBI y dispersión del IPM. Para ello se estima la ecuación 6.

Tabla 5 - Convergencia- y crecimiento económico

\begin{tabular}{ll}
\hline $\begin{array}{l}\text { Dependiente: desvío } \\
\text { estándar logaritmo IPM }\end{array}$ & $2004-2016$ \\
\hline$d$ & $\begin{array}{l}0.0013^{* *} \\
(0.0007)\end{array}$ \\
\hline Constante & 0.2505 \\
\hline $\mathrm{N}$ & 448 \\
\hline $\mathrm{R}^{2}$ & 0.05 \\
\hline
\end{tabular}

Fuente: elaboración propia en base a EPH-INDEC

${ }^{*}$ significativo al $10 \%,{ }^{* *}$ significativo al $5 \%,{ }^{* * *}$ significativo al $1 \%$

Se observa que no es posible rechazar, al 5 por ciento, la hipótesis que el crecimiento económico, medido a través del PBI, contribuya al incremento de las disparidades regionales de pobreza en Argentina. Este resultado de ninguna manera debe ser entendido como una asociación negativa entre crecimiento y pobreza, sino sólo en términos de crecimiento y disparidades regionales de pobreza.

\section{Conclusiones}

En este trabajo se ha analizado la relación entre disparidades territoriales de pobreza multidimensional y crecimiento económico para el caso de Argentina. Los resultados sugieren la existencia de una relación positiva. Esto es, los años de mayor crecimiento económico (2003-2007) presentaron una tendencia divergente en los niveles de pobreza multidimensional entre los principales aglomerados urbanos del país (mayores disparidades). En cambio, en los años en los que el crecimiento económico se desaceleró progresivamente-entre 2008-2011 y 2012-2016-, tuvo lugar un proceso de convergencia en los niveles de pobreza (menores disparidades). Lo anterior no debe confundirse con el vínculo entre crecimiento y reducción de la pobreza, cuya relación también es positiva. Esto es, en los 
años de mayor crecimiento económico se observan mayores reducciones de la pobreza.

Las estimaciones de convergencia- $\beta$ absoluta sugieren que entre 20042016 tuvo lugar un proceso de convergencia entre aglomerados urbanos. Sin embargo, al desagregar en subperíodos, se observa que en aquel de mayor crecimiento (2004-2007) existió una dinámica de divergencia ( $\beta$ significativo y positivo), esto es, los aglomerados menos pobres reducían más rápido la pobreza. Luego, efectivamente tuvo lugar una dinámica convergente-aglomerados más pobres reducen más rápido sus niveles de pobreza -y lo cual se acentuó en el último subperíodo (2012-2016). Las estimaciones de convergencia- replican aquellas de convergencia- $\beta$ y le otorgan al trabajo una mayor robustez.

A lo largo de este trabajo hemos empleado a una medición multidimensional de la pobreza, la cual incluye, pero excede la dimensión monetaria, incorporando las dimensiones de educación, acceso a servicios, vivienda y empleo y seguridad social. En este sentido, el trabajo se apartó de la literatura tradicional de convergencia de ingresos y propuso un análisis de la dinámica del bienestar (o la falta de este) en un espacio multidimensional.

La regularidad empírica entre crecimiento y disparidades territoriales de pobreza evidencia la existencia de un dilema para los hacedores de política: intentar favorecer el crecimiento económico para reducir la pobreza a costa de aumentar las disparidades territoriales o, aceptar menores niveles de crecimiento promoviendo una mayor igualdad territorial en pobreza. Lógicamente, la solución es compleja e involucra la consideración de diversos factores para lograr un crecimiento inclusivo.

Se debe prestar especial atención la situación de los aglomerados más rezagados. Típicamente en este grupo se incluyen a aquellos del Norte Argentino. Una esperanza de vida significativamente menor a la del resto del país, mayores tasas de mortalidad infantil y desnutrición parecen sugerir la consideración prioritaria de esta región.

A su vez, no debe olvidarse que un mayor crecimiento económico se asocia a mayores reducciones de la pobreza. Por ello, las acciones de política macroeconómica deben promover el crecimiento, pero propiciando aquellas medidas que permitan atenuar las dinámicas divergentes entre aglomerados.

Lógicamente sería deseable contar con datos con mayor cobertura geográfica, que incluyeran pequeños aglomerados urbanos y zonas rurales, de modo de robustecer los resultados aquí hallados. 


\section{Anexo}

Tabla 6 - Indicadores seleccionados por región

\begin{tabular}{|c|c|c|c|c|c|}
\hline Región & Población $^{\mathrm{a}}$ & $\begin{array}{l}\text { \% Población } \\
\text { País }\end{array}$ & \% PBI País & $\begin{array}{l}\text { \% Export. } \\
\text { País b }\end{array}$ & $\begin{array}{l}\text { \% Empleos } \\
\text { Registrados } \\
\text { País }{ }^{c}\end{array}$ \\
\hline NEA & $3,679,609$ & 9.17 & 3.75 & 1.7 & 4.42 \\
\hline NOA & $4,911,412$ & 12.24 & 6.46 & 7 & 7.44 \\
\hline Cuyo & $2,852,294$ & 7.11 & 6.41 & 5.7 & 6.07 \\
\hline Centro & $26,573,593$ & 66.24 & 75.07 & 73.4 & 75.57 \\
\hline Sur & $2,100,188$ & 5.24 & 8.31 & 8.4 & 6.50 \\
\hline Argentina & $40,117,096$ & 100 & 100 & 100 & 100 \\
\hline
\end{tabular}

Fuente: González (2019)

a Cálculos propios en base al Censo Nacional de Población, Hogares y Viviendas 2010.

$\mathrm{b}$ No se incluyen las exportaciones de origen indeterminado ni aquellas mercaderías sujetas a un régimen de admisión temporal.

c Cálculos propios para el IV Trimestre de 2017.

Tabla 7 - Hogares con NBI y mortalidad infantil por región

\begin{tabular}{llll}
\hline Región & $\begin{array}{l}\text { Tasa de Mortalidad } \\
\text { infantil }^{\mathrm{a}}\end{array}$ & $\begin{array}{l}\text { \% de hogares } \\
\text { con NBI 2001 }\end{array}$ & $\begin{array}{l}\text { \% de hogares } \\
\text { con NBI 2010 }\end{array}$ \\
\hline NEA & 11.60 & 25.48 & 16.79 \\
\hline NOA & 11.12 & 23.58 & 15.60 \\
\hline Cuyo & 8.92 & 13.37 & 8.23 \\
\hline Centro & 9.21 & 11.82 & 7.37 \\
\hline Sur & 8.40 & 13.71 & 8.75 \\
\hline Argentina & 9.67 & 14.32 & 9.13 \\
\hline
\end{tabular}

Fuente: González (2019)

${ }^{a}$ No incluye a los nacidos en otros países o cuyo lugar de nacimiento no se haya especificado.

${ }^{\mathrm{b}}$ No incluye a personas que habitan en viviendas colectivas.

Tabla 8 - Indicadores seleccionados sistema de salud público

\begin{tabular}{llllll}
\hline & $\begin{array}{l}\text { Gasto per } \\
\text { cápita } \\
\text { anual }^{\mathrm{a}}\end{array}$ & $\begin{array}{l}\text { Habitantes } \\
\text { por } \\
\text { médico }\end{array}$ & $\begin{array}{l}\text { Habitantes } \\
\text { por cama }\end{array}$ & $\begin{array}{l}\text { Personas sin } \\
\text { cobertura de } \\
\text { salud en } \%\end{array}$ & $\begin{array}{l}\text { Esperanza } \\
\text { de vida al } \\
\text { nacer }^{c}\end{array}$ \\
\hline NEA & 378 & 600 & 309 & 50.9 & 73.85 \\
\hline NOA & 461 & 491 & 291 & 44.2 & 75.06 \\
\hline Argentina & 395 & 331 & 262 & 36.1 & 75.34 \\
\hline
\end{tabular}

Fuente: González (2019)

${ }^{a}$ Gasto per cápita anual provincial en atención de la salud, en pesos corrientes de 2008.

${ }^{\mathrm{b}}$ Personas sin cobertura de salud que habitan en viviendas particulares.

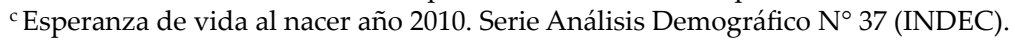




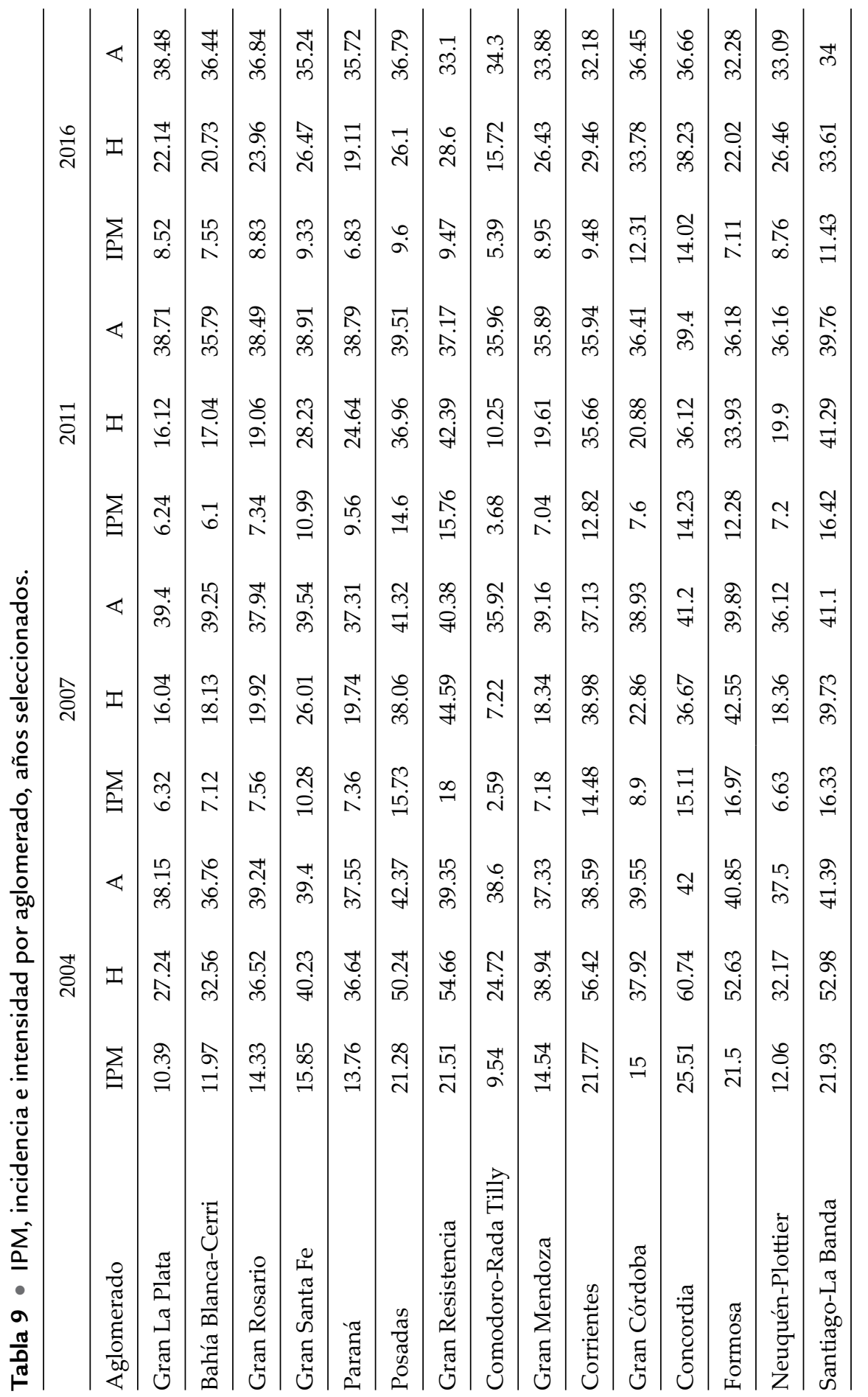




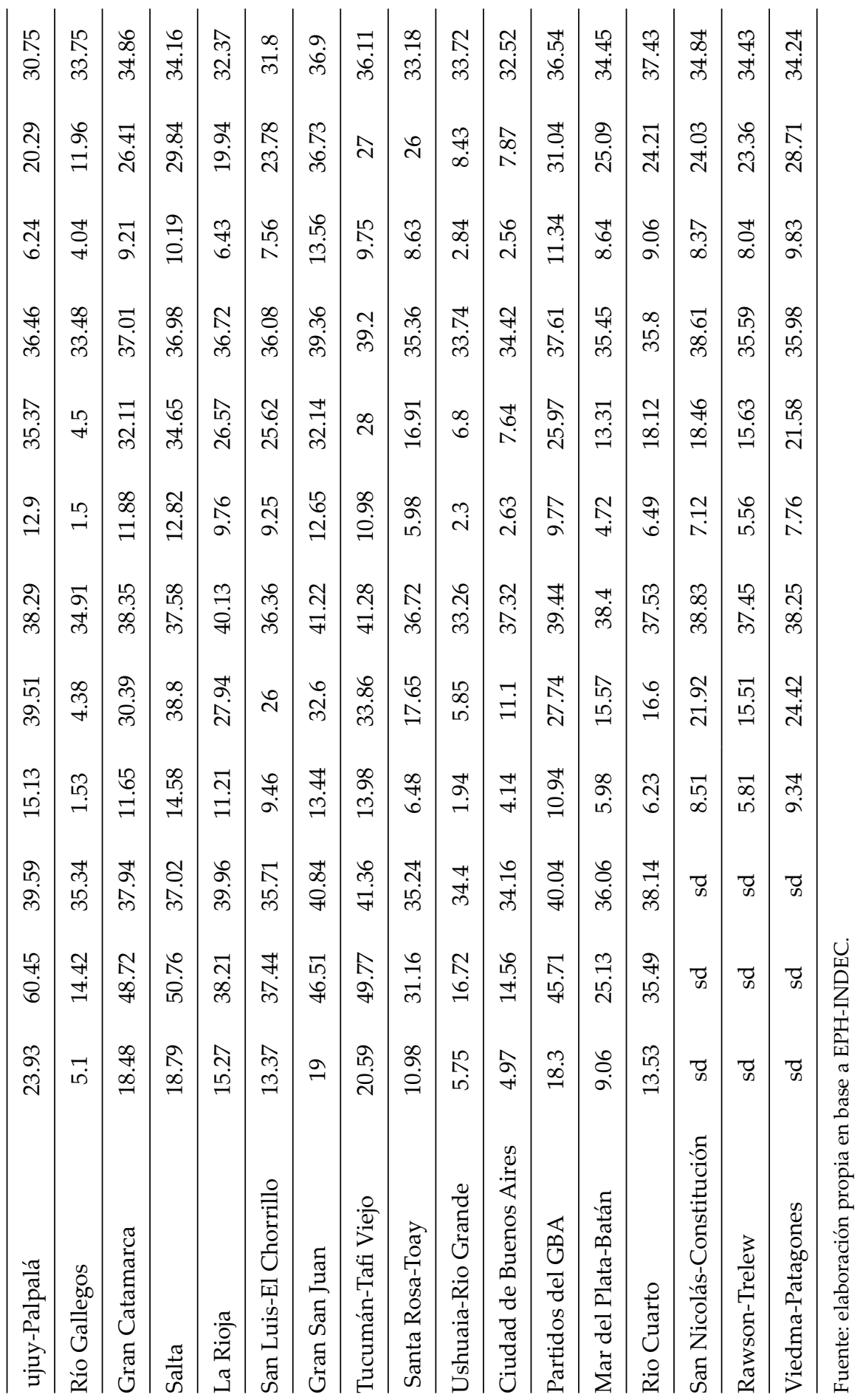




\section{AGRADECIMIENTOS}

Se agradecen los comentarios del Dr. Juan Cruz Fernández.

FERNANDO ANTONIO IGNACIO GONZÁLEZ Magister en economía. Becario doctoral en Instituto de Investigaciones Económicas y Sociales del Sur en el Instituto de Investigaciones Económicas y Sociales del Sur, UNS-CONICET. Email: faigonzalez@iiess-conicet.gob.ar

MARIA EMMA SANTOS Doctora en economía, Investigadora en Instituto de Investigaciones Económicas y Sociales del Sur UNS-CONICET y Profesora en Departamento de Economía, UNS. Email: msantos@uns.edu.ar

SILVIA LONDON Doctora en economía Investigadora en Instituto de Investigaciones Económicas y Sociales del Sur UNS-CONICET y Profesora en Departamento de Economía, UNS. email: slondon@uns.edu.ar

\section{NOTES}

1. Las demás regiones existentes son el Centro (Córdoba, Santa Fe, Entre Ríos, Ciudad de Buenos Aires y Provincia de Buenos Aires), Cuyo (Mendoza, San Juan y San Luis) y Sur (Rio Negro, Neuquén, La Pampa, Santa Cruz, Chubut y Tierra del Fuego).

2. Entre 2003-2015 gobernó el Frente para la Victoria (partido de centro-izquierda) mientras que entre 2015-2019 lo hizo Cambiemos (centro-derecha).

3. La densidad de habitantes (personas por $\mathrm{km}^{2}$ ), hacia 1,500, es considerada una medida de prosperidad para la época dado que solo urbanizaciones con alta productividad agrícola y redes de transporte desarrolladas podían sostener grandes aglomeraciones (Acemoglu et al., 2002, p 1232).

4. Instituciones extractivas son aquellas que tienden a concentrar el poder en una pequeña élite local, acompañado de un alto riesgo de expropiación para la mayor parte de la población (Acemoglu et al., 2002, p. 1235).

5. La modalidad continua de la EPH inicia en el segundo semestre de 2003 y por ello no se cuenta con información referida al primer semestre de ese año. Respecto al faltante en III-2007, trabajadores del INDEC no llevaron a cabo las encuestas de hogares de ese período en el marco de reclamos por la intervención del organismo. Desde ese año y hasta 2015 el INDEC se encontró intervenido y en este contexto decidió no difundir la información de la EPH del segundo semestre de 2015. La normalización del organismo en 2016, no fue inmediata y por ello tampoco se difundieron los resultados de I-2016.

6. En Córdoba se relevan los aglomerados Gran Córdoba y Rio Cuarto. En Santa Fe, Gran Santa Fe y Gran Rosario. En Entre Ríos, Gran Paraná y Concordia. En Chubut, Rawson-Trelew y Comodoro Rivadavia-Rada Tilly. En Buenos Aires, Bahía Blanca-Cerri, Gran La Plata, Mar del Plata-Batán y San Nicolás-Villa Constitución. Los partidos bonaerenses limítrofes a la Ciudad de Buenos Aires, se incluyen en el aglomerado Gran Buenos Aires 
7. El informe se refiere a aspectos de diseño muestral de la EPH, modificado en 2013, debido a falta de supervisión adecuada en la realización de las encuestas, creciente no respuesta en variables muy importantes tales como montos y fuentes de ingresos, ausencia de documentación técnica que explicite los criterios conceptuales y operativos utilizados por ejemplo para la imputación de ingresos faltantes, entre otras cosas.

8. El cálculo surge de despejar $n$ de la siguiente ecuación: e-vn=0,5

\section{REFERENCIAS}

Acemoglu, D., Johnson, S., \& Robinson, J. (2002). Reversal of fortune: Geography and institutions in the making of the modern world income distribution. Quarterly Journal of Economics 117(4), 1231-1294. doi: 10.1162/003355302320935025.

Alesina, A., \& Perotti, R. (1996). Income distribution, political instability, and investment. European Economic Review 40(6), 1203-1228. doi: 10.1016/00142921(95)00030-5.

Alkire, S., \& Foster, J. (2007, revisado en 2008). Recuento y medición multidimensional de la pobreza (Documento de trabajo OPHI No. 7). Descargado de https://ophi .org.uk/publications/ophi-working-papers/.

Alkire, S., \& Foster, J. (2011). Understandings and misunderstandings of multidimensional poverty measurement (Documento de trabajo OPHI No. 43). Descargado de https://ophi.org.uk/publications/ophi-working-papers/.

Alkire, S., \& Santos, M. (2010). Acute multidimensional poverty: A new index for developing countries (Documento de trabajo OPHI No. 38). Descargado de https:// ophi.org.uk/publications/ophi-working-papers/.

Alvaredo, F., \& Gasparini, L. (2013). Recent trends in inequality and poverty in developing countries (Documento de trabajo CEDLAS No. 151). Descargado de http:// www.cedlas.econo.unlp.edu.ar/wp/wp-content/uploads/doc_cedlas151.pdf.

Aráoz, M., \& Nicolini, E. (2015). Persistence vs. reversal and agglomeration economies vs. natural resources: Regional inequality in Argentina in the first half of the twentieth century (Documento de trabajo Instituto Figuerola de Historia y Ciencias Sociales, Universidad Carlos III de Madrid No. 15). Descargado de https://e-ar chivo.uc3m.es/bitstream/handle/10016/20846/wp1505.pdf?sequence=1.

Aráoz, M., \& Nicolini, E. (2016). The evolution of regional GDPs in Argentina during the period of globalization at the end of the 19th and beginning of the 20th century. Anales de la LI Reunión Anual de la Asociación Argentina de Economía Política, Tucumán. Descargado de https://aaep.org.ar/anales/buscador.php.

Atkinson, T. (2015). Inequality: what can be done? Cambridge: Harvard University Press.

Banco Mundial. (2020). Serie Producto Bruto Interno. Descargado de https://datos .bancomundial.org/indicator/NY.GDP.PCAP.KN?locations=AR.

Bernard, A., \& Jones, C. (1996). Productivity and convergence across US states and industries. Empirical Economics 21(1), 113-135. doi: 10.1007/BF01205496.

Brida, G., Garrido, N., \& London, S. (2013). Estudio del desempeño económico regional: El caso argentino. Cuadernos de Economía 32(60), 437-466. 
Brida, G., Sanchez, E., \& Segarra, V. (2020). Clustering and regime dynamics for economic growth and income inequality. Structural Change and Economic Dynamics 52, 99-108. doi: 10.1016/j.strueco.2019.09.010.

Capello, M., Figueras, A., Freille, S., \& Moncarz, P. (2013). The role of federal transfers in regional convergence in human development indicators in Argentina. Investigaciones regionales $27,33-63$.

Figueras, A., Cristina, D., Blanco, V., \& Capello, M. (2014). Un aporte sobre el debate de la convergencia en Argentina: la importancia de los cambios estructurales. Revista Finanzas y política económica 6(2), 287-316.

FMI. (2015). Causes and consequences of income inequality: A global perspective (Discussion note FMI No. 15/13). Descargado de https://www.imf.org/external/pubs/ $\mathrm{ft} / \mathrm{sdn} / 2015 / \mathrm{sdn} 1513$.pdf.

Garrido, N., Marina, A., \& Sotelsek, D. (2001). Convergencia económica en provincias argentinas (1970-1995). Estudios de Economía Aplicada 20(2), 403-421.

González, F. (2019). Pobreza multidimensional urbana en Argentina: Un análisis de las disparidades entre el Norte Grande y Centro-Cuyo-Sur (2003-2016). Tesis de Maestría en Economía, Universidad Nacional del Sur. Descargado de http://repo sitoriodigital.uns.edu.ar/bitstream/123456789/4757/1/Tesis percent20Fer.pdf.

González, F., \& Santos, M. (2020). Pobreza multidimensional urbana en Argentina: ¿Reducción de las disparidades entre el Norte Grande Argentino y Centro-Cuyo-Sur? (2003-2016). Cuadernos de Economía 39(81), 795-822.

INDEC. (2003). Series históricas de pobreza y desempleo. Descargado de https://www .indec.gob.ar/indec/web/Institucional-Indec-InformacionDeArchivo-2

INDEC. (2016a). Incidencia de la pobreza y la indigencia en 31 aglomerados urbanos: resultados del segundo trimestre de 2016. Informe de prensa INDEC. Descargado de https://www.indec.gob.ar/uploads/informesdeprensa/eph_pobreza_01_16 .pdf.

INDEC. (2016b). Mercado de trabajo: Principales indicadores. Segundo trimestre de 2016. Consideraciones sobre la revisión, evaluación y recuperación de la Encuesta Permanente de Hogares (EPH). Anexo Informe de Prensa. Descargado de https:// www.indec.gob.ar/ftp/cuadros/sociedad/anexo_informe_eph_23_08_16.pdf.

INDEC. (2018). Series trimestrales de oferta y demanda Globales. Años 2004 a 2018. Descargado de https://www.indec.gob.ar/nivel4_default.asp?id_tema_ $1=3 \&$ \&id_tema_2=9\&id_tema_3 $=47$.

Kong, J., Phillips, P., \& Sul, D. (2017). Weak б-convergence: Theory and applications (Documento de discusión Cowles Foundation for research in Economics-Yale University No. 2072). Descargado de https://cowles.yale.edu/sites/default/ files/files/pub/d20/d2072.pdf.

Krugman, P. (2012). End this depression now! New York: W. W. Norton \& Company. Maloney, W., \& Valencia Caicedo, F. (2012). The persistence of (Subnational) fortune geography, agglomeration, and institutions in the New World (Documento de trabajo Banco Mundial No. 6187). Descargado de https://openknowledge.world bank.org/handle/10986/12047?locale-attribute=es.

OCDE. (2015). In it together: Why less inequality benefits all? Paris: OCDE Publishing. Piketty, T. (2014). Capital in the 21st Century. Cambridge: Harvard University Press. 
Porto, G. (1994). Convergencia y política económica: Algunos resultados para provincias argentinas. Anales de la XXIX Reunión Anual de la Asociación Argentina de Economía Política, La Plata. Descargado de https://aaep.org.ar/anales/busca dor.php.

Qureshi, Z. (2017). Trends in income inequality: Global, inter-country, and within countries (Informe Brookings Institution No. 12). Descargado de https://www .brookings.edu/wp-content/uploads/2017/12/global-inequality.pdf.

Ramón-Berjano, C. (2004). Socio-economic disparities in Argentina y Brazil. Tesis de doctorado en economía de la University of London.

Santos, M., Dabús, C., \& Delbianco, F. (2019). Growth and poverty revisited from a multidimensional perspective. Journal of Development Studies 55(2), 260-277. doi: 10.1080/00220388.2017.1393520.

Santos, M., \& Villatoro, P. (2018). A multidimensional poverty index for Latin America. The Review of Income and Wealth 64(1), 52-82. doi: 10.1111/roiw.12275.

Santos, M., Villatoro, P., Mancero X., \& Gerstenfeld, P. (2015). A multidimensional poverty index for Latin America (Documento de trabajo OPHI No. 79). Descargado de https://ophi.org.uk/publications/ophi-working-papers/.

Sen, A. (1985). A sociological approach to the measurement of poverty: A reply to Professor Peter Townsend. Oxford Economic Papers 37, 669-676.

Sen, A. (2009). The idea of justice. Cambridge: Harvard University Press.

Talassino, M. (2015). Producto bruto geográfico de 1946 en las provincias argentinas: Una estimación preliminar. Anales de la L Reunión Anual de la Asociación Argentina de Economía Política, Salta. Descargado de https://aaep.org.ar/anales/ buscador.php.

Talassino, M. (2017). Convergencia regional argentina: Análisis exploratorio y econométrico espacial basado en el Producto geográfico departamental. Anales de la LII Reunión Anual de la Asociación Argentina de Economía Política, Bariloche. Descargado de https://aaep.org.ar/anales/buscador.php.

Utrera, G., \& Koroch, J. (1998). Convergencia: Evidencia empírica para las provincias argentinas (1953-1994). Anales de la XXXIII Reunión Anual de la Asociación Argentina de Economía Política, Mendoza. Descargado de https://aaep.org .ar/anales/buscador.php.

\title{
Convergence and development in urban Argentina (2003-2016)
}

\section{Fernando Antonio Ignacio González, Maria Emma Santos \& Silvia London}

\begin{abstract}
This article analyses the recent evolution of territorial disparities in Argentina, measured by a Multidimensional Poverty Index, by carrying out a formal convergence analysis between agglomerates. In particular, the existence of absolute $\beta$-convergence and $\sigma$-convergence is tested. The information comes from the microdata of the Permanent Household Survey. The results suggest that, although an end-to-end analysis of the period shows a decrease in poverty accompanied by a
\end{abstract}


reduction in territorial disparities between the main urban agglomerates in Argentina, when disaggregating by subperiods, it is evident that in periods of economic growth (essentially from 2003 to 2009), territorial disparities increased. Conversely, in periods of stagnation, these disparities decrease.

Keywords: economic growth, household, microdata, multidimensional poverty, territorial disparities

\section{Convergence et développement dans l'Argentine urbaine (2003-2016)}

\section{Fernando Antonio Ignacio González, Maria Emma Santos \& Silvia London}

Résumé: Ce travail analyse l'évolution récente des disparités territoriales en Argentine, mesurée à partir d'un Indice multidimensionnel de la pauvreté, sur la base d'une étude de la convergence entre les agglomérations urbaines. Il teste en particulier l'existence de la beta convergence absolue et de la sigma convergence. Les informations proviennent de microdonnées de l'Enquête permanente des ménages. Bien qu'une analyse ponctuelle de la période montre une réduction générale de la pauvreté accompagnée d'une réduction des disparités territoriales entre les principales agglomérations urbaines d'Argentine, la désagrégation par sous-périodes permet d'observer que pendant les périodes de croissance économique (essentiellement de 2003 à 2009), les disparités territoriales se sont accrues alors qu'en période de stagnation elles se sont réduites.

Mots-clés: croissance économique, disparités territoriales, foyers, microdonnées, pauvreté multidimensionnelle. 